\title{
Radar sensor for an autonomous antarctic explorer
}

\author{
Alex Foessel, Dimi Apostolopoulos, William "Red" Whittaker \\ Robotics Institute, Carnegie Mellon University, \\ 5000 Forbes Avenue, Pittsburgh, PA 15213
}

\begin{abstract}
The localization and identification of antarctic meteorites is a task of great scientific interest and with implications to planetary exploration. Autonomous search for antarctic meteorites presents a profound technical challenge. Ground Penetrating Radar (GPR) holds the prospect to safeguard antarctic robot from terrain dangers and detect subsurface objects.

In January 1998, we validated a $500 \mathrm{MHz}$ GPR sensor as part of as field robotic technology demonstration at Patriot Hills, Antarctica. We deployed the sensor from a sled and integrated with position and attitude instruments to perform field measurements. Data was acquired under different conditions and in multiple locations. The radar detected hidden crevasses from $50 \mathrm{~cm}$. distance, thus showing its merit as a rover safeguarding device. It also localized $5 \mathrm{~cm}$. rocks in snow and ice. Moreover, the radar data was used to characterize snow/ice/bedrock stratigraphy. GPS position measurements enabled ground truth and mapping of the location of hazards and interesting subsurface objects and features.
\end{abstract}

This paper summarizes the experiments performed and discusses on the utility of the GPR sensor for autonomous antarctic robots.

Keywords: Antarctica, GPR, robotic explorers, meteorite search

\section{INTRODUCTION}

Antarctica presents a close analog to planetary exploration with regards to environmental survival, communication, power, navigation, remote operations, and scientific agenda. An ideal context for robotic antarctic demonstrations with relevance to planetary exploration is the search for antarctic meteorites. Through tireless investigation in the harsh antarctic environment and using computer sensing to search above and below the ice surface, robots will explore regions of Antarctica to find otherwise undetected meteorites. The use of robots will augment the human search for meteorites by working full-day cycles in the deep cold, and by detecting surface meteorites obscured to the human eye by blowing or drifting snow.

A robot that could sense objects buried in snow would contribute to a human meteorite search team, by making the search task complete. A mobile robot could perform a systematic survey of blue ice covered by snow, detecting and recording the position of the findings while the human team performs visual search. In a few years, robots could find meteorites not visible to humans, overlooked by humans, or in areas challenging to the human search. The

Further author information:

A.F. (correspondence): Email: afoessel@ cmu.edu; WWW: http://www.cs.cmu.edu/ afoessel

D.A.: Email: da1v@frc.ri.cmu.edu

W.W.: Email: red@frc.ri.cmu.edu 
robot would build the current snow patch map while systematically scanning the search area. The machine report would contain the position of the hidden targets for later human retrieval.

Several meteorite findings in a small area indicate the existence of meteorite concentration. In such a site, more meteorites are expected to be found if careful and systematic search is conducted. However, snowdrift patches could partially cover the ice field, leaving meteorites hidden from human sight. The cost of marking areas as searched and a second visit to complete the search generally precludes the meteorite harvest completion.

To achieve detection of subsurface meteorites GPR is a preferred sensor. By analyzing the electromagnetic signal reflected by discontinuity in the dielectric properties of the media, buried objects can be detected. Such a sensor could also be used to detect non visible voids in the ground and thus work as a robotic safeguarding device. In January 1998, a $500 \mathrm{MHz}$ GPR was deployed as part of as field robotic technology demonstration at Patriot Hills, Antarctica. The sensor was deployed from a sled and integrated with position instruments to perform field measurements. This paper describes the approach used to detect subsurface meteorites in Antarctica and critiques the results.

\section{SENSOR DEPLOYMENT IN ANTARCTICA}

From December 29th, 1997 to January 22nd, 1998, a team of scientists and roboticists visited Patriot Hills, located near $80^{\circ} \mathrm{S}$ and $81^{\circ} \mathrm{W}$ in Ellsworth Land, Antarctica. The purpose of the expedition was to validate mobile robot perception technologies in a polar setting and sensors for detection of meteorites. This was the first step towards comprehensive agenda with an autonomous robot equipped with meteorite detection sensors. No meteorites were found ${ }^{1}$, nevertheless science sensors were evaluated on "planted" meteorite samples brought to the site.

The fact that no meteorites were found is believed to be due to the temperature and altitude conditions of the area. These conditions suggest that existing meteorites would be buried in ice or snow ${ }^{1}$, thus providing additional motivation for undersurface probing research.

Research with the GPR sensor was conducted in areas of blue ice. Rocks were planted in the ice and under snowdrifts. An icefield with ice cracks and a man made void served as examples of crevasses. Also, a suitable ice area with snow patches was selected for the detection of snowdrift layers on ice.
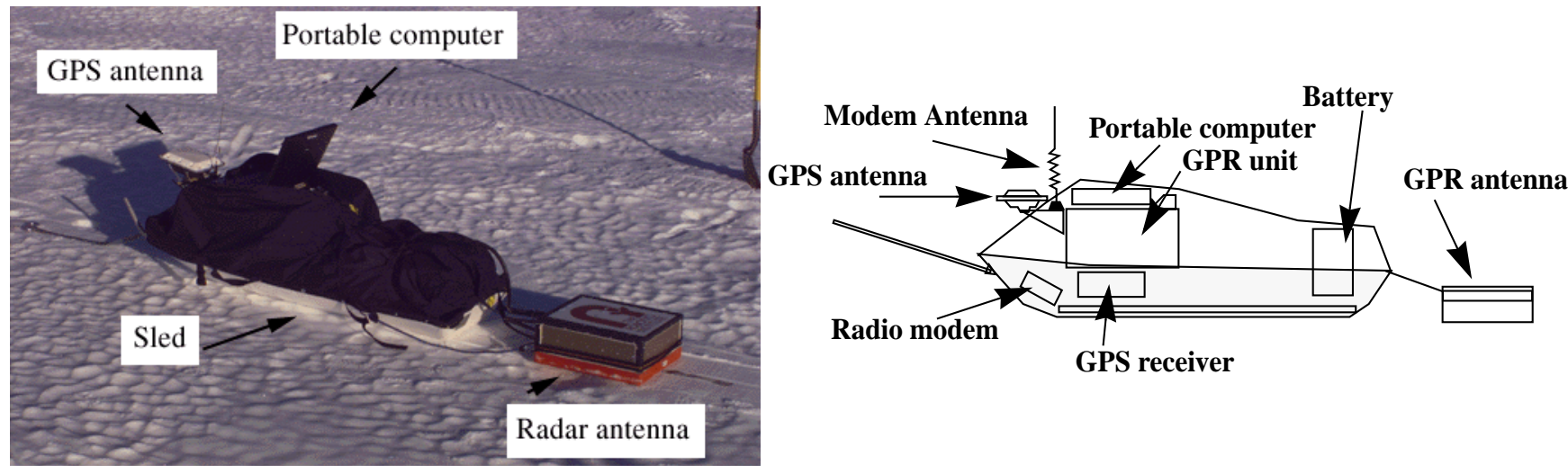

FIGURE 1. Radar sensor deployed in Patriot Hills, Antarctica, January 1998. 


\subsection{GPR sensor configuration for meteorite search use}

The ground penetrating radar used was a GSSI Subsurface Interface Radar (SIR) System 3. This is a portable broadband pulse radar capable of detecting and digitally recording the position and depth of objects buried in dielectric materials. It operates with one antenna with a center frequency of $500 \mathrm{MHz}$. The original paper inscriptor was removed from the radar main unit, and a portable computer was connected instead. An analog connection transmitted the signals to the A/D PCMCIA card. The data was acquired using a graphical based interface and stored on the portable computer's hard disk. A GPS receiver was also connected to the computer. This GPS receiver has real time differential correction capability with accuracy better than $10 \mathrm{~cm}$. The position information was used to correct the underground radar profiles for speed variations. The GPS receiver, the radar and the portable computer were mounted in a small human pulled sled, as shown in Figure 1. A modem antenna is used for transmission of differential corrections to the sled, providing decimeter accuracy position information for each measurement.

During this expedition, the data acquisition was controlled manually. The data conditioning and feature detection was visual. A custom graphical user interface (GUI), shown in Figure 2, was developed for easy data acquisition operations. The GPR, GPS and portable computer integrated on the sled performed the acquisition of GPR data with associated time and position information automatically, leaving the operator free to pull the sled and cover the area of interest.

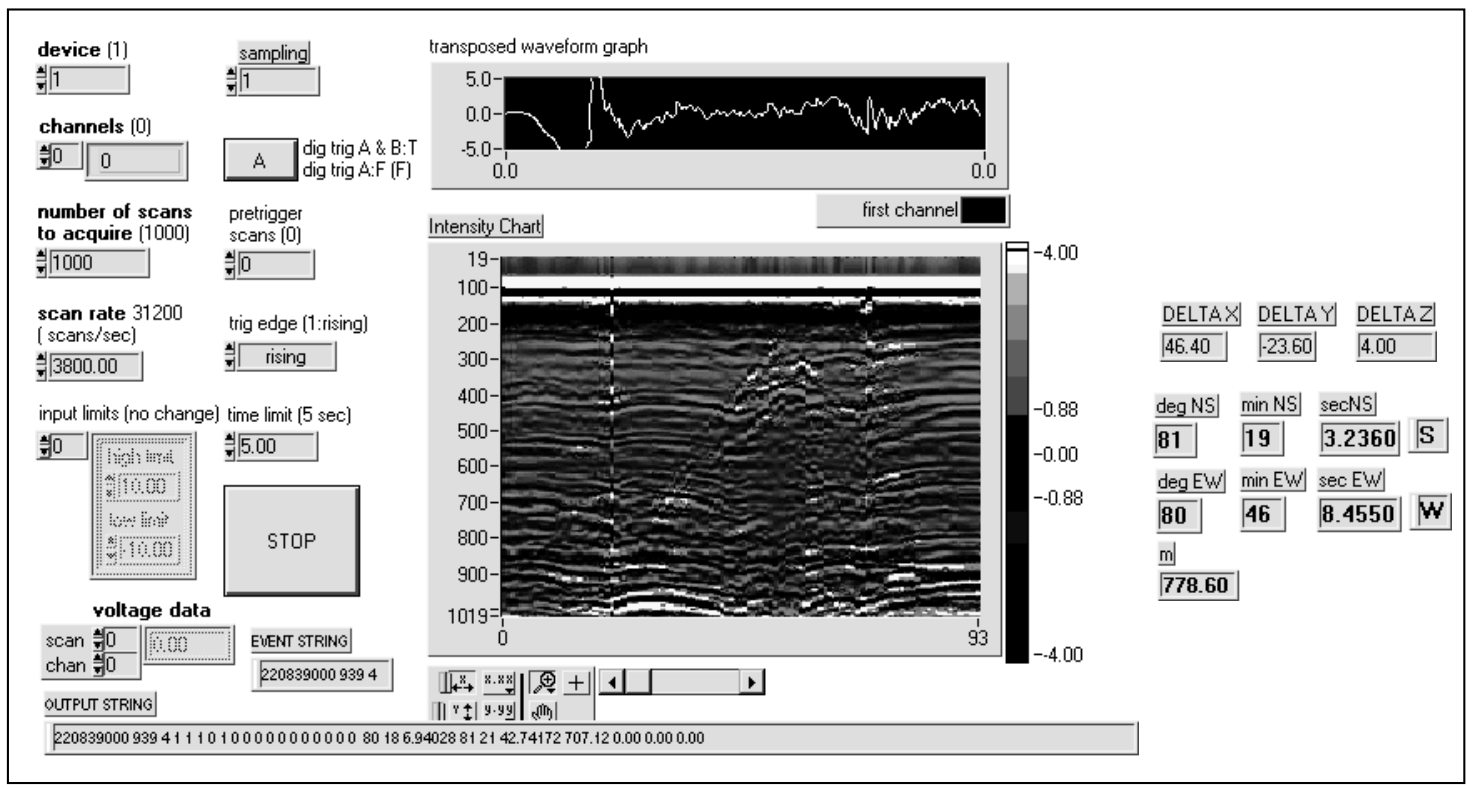

FIGURE 2. Graphical interface for GPR data acquisition

The GPR data is displayed on a two dimensional graph. The vertical axis shows the time of the microwave energy travelling into the ground and being reflected by the dielectric discontinuity. The gray intensity shows the level of the signal at that particular time. Each trace is displayed sequentially along the horizontal axis. The display scrolls horizontally when new data is acquired. This presents a convenient way to adjust the gain controls on the GPR console in real time until a proper display is obtained.

The display also shows the sled position as estimated by the GPS receiver with respect to Earth coordinates and with respect to local coordinates (origin: the differential fixed base station). The GPS was tested to understand its usefulness as a ground truth device. Ground truth is required by most GPR analysis techniques for correcting the 
variations of the antenna speed, which is done off-line. In a static test, the differential GPS setting proved to be within $5 \mathrm{~cm}$ of the initial reading, accurate enough to build maps and correct for the horizontal speed variations.

The acquisition rate was limited by the display and storage speed of the portable computer. The system performed at $2 \mathrm{~Hz}$. This proved to be insufficient for a walking speed $(\sim 100 \mathrm{~cm} / \mathrm{s})$ providing only two measurements per meter. A faster system is needed to provide the necessary sampling rate to assure the detection of small targets.

\subsection{Buried object detection}

The test rocks were placed at depths ranging from $5 \mathrm{~cm}$ to $50 \mathrm{~cm}$. Although the radar is capable of providing information of deeper objects, they are not of interest in this research because no finding below $50 \mathrm{~cm}$ would be retrieved by a human team searching for meteorites in antarctic conditions.

Although meteorites are found in sizes ranging from an olive to a water melon, only targets from a $4 \mathrm{~cm}$. to $30 \mathrm{~cm}$ in diameter were selected for testing. The wavelength of the radar $(60 \mathrm{~cm}$ in air, $\sim 30 \mathrm{~cm}$ in ice) makes the detection of rocks smaller than $7.5 \mathrm{~cm}$ (a quarter of the wavelength) difficult.

It is important to note that GPR can detect an object but does not provide any info on its composition. In case of meteorite detection, object size and shape may be the only indicators of whether a rock is a meteorite or not, and if it should be retrieved.

To test GPR, seven rocks were placed at $1 \mathrm{~m}$ separation in a line. The position of the rocks was well known and the sled dragged the GPR antenna over the line several times. Figure 3 shows one rock that is buried and the corresponding radar signature. Note the two strong signals at the two ends of the graphs: they correspond to metallic bars that were laid at the extremes of the line of rocks to provide a point of reference. The distance between the bars is $8 \mathrm{~m}$. The square shows the signal that indicates the presence of a target (the white curve was manually superimposed to illustrate the shape of the signal).

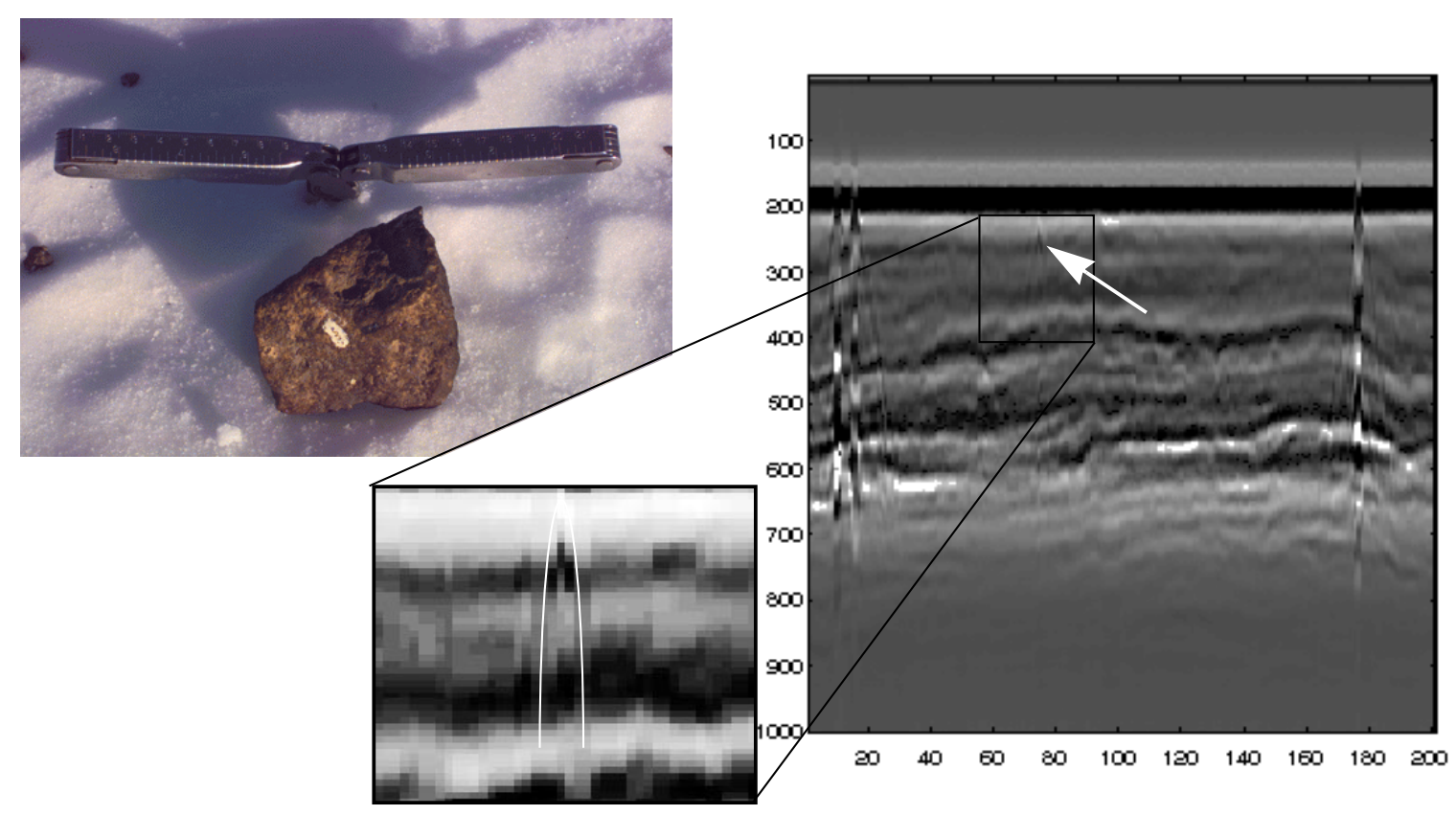

FIGURE 3. Rock and the GPR signature of buried rock. 
The wide beam of the radar produces a hyperbolic signature of a point reflector when the antenna has a linear motion. As the antenna approaches the buried object, the slant distance between the object and the antenna decreases. This distance reaches a the minimum when the antenna is right above the object and increases as the antenna moves away.

The half power pattern of the GPR antenna shows a beam width of 90 degrees (45 degrees to each side of the vertical). The Figure 3 profile detail has a hyperbola that approximates the target reflected energy spread. There are techniques that address this phenomena concentrating and sharpening the images and facilitating the visualization and potential automatic detection of this targets ${ }^{2}$.

In both test areas, the largest rocks are clearly visible. Rocks smaller than $5 \mathrm{~cm}$. are not visible with simple observation of the radar data. Furthermore, GPR at $500 \mathrm{MHz}$ has been used to probe to depths of a few tens of meters in to the ice ${ }^{3}$. Since we wish to examine smaller objects and only depths of $50 \mathrm{~cm}$, a higher frequency GPR should be used.

\subsection{Void and crevasse detection}

Crevasses are a common danger for antarctic exploration. The most dangerous are those hidden by a bridge of snow that collapses under weight. Airborne crevasse detection has been performed on Antarctic routes ${ }^{4} 5$. Previous work shows the feasibility of detection of voids and caves ${ }^{6}$.

An ice field with ice cracks was selected to safely evaluate the crevasse detection potential. The ice cracks were between 10 to $30 \mathrm{~cm}$ wide and filled with snow. The sensor was dragged over them to evaluate the potential early detection. A man made void with spherical shape ( $2.5 \mathrm{~m}$ diameter) was used to test the detection of wide crevasses covered by bridges of snow. The void was probed in a rectangular pattern from one side to the other and the shape was recorded for reference.

Figure 4 shows one of the many profiles, this one cuts through the center of the void. The segmented circle shows the approximate shape of the void under the snow.

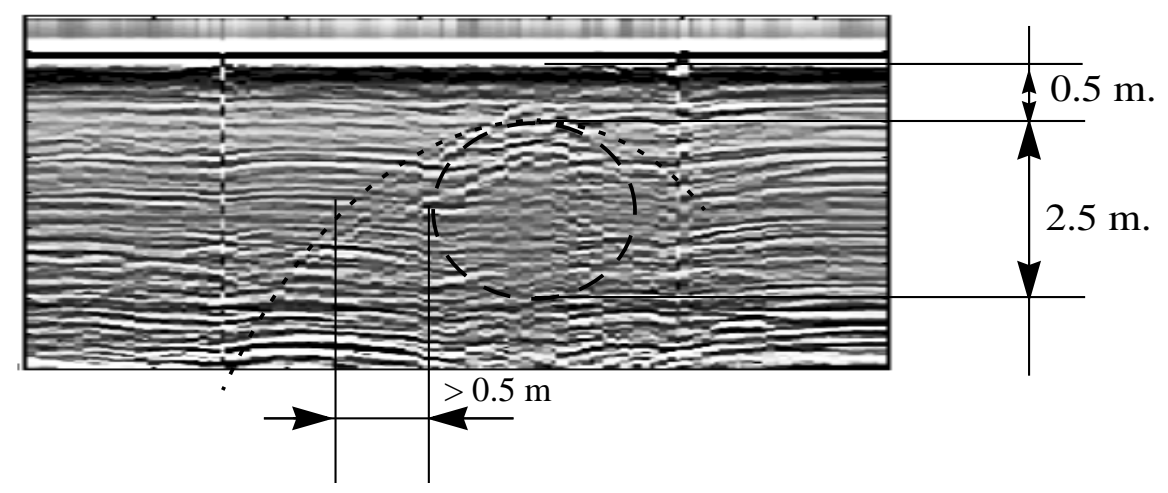

FIGURE 4. Profile over an artificial void in the ice.

An interesting fact is that the void was detected $50 \mathrm{~cm}$ before the antenna was above the edge of the void. The hypothesis is the wide beam extends ahead of the antenna providing early detection. This feature is of most importance for GPR as a safeguarding sensor. 


\subsection{Ice/snow layer detection}

Meteorites are usually found on blue ice fields. However, the wind drifts snow over them in continuously changing patterns of snow patches covering blue ice. As a result, a meteorite concentration can not be completely harvested because these seasonal snow patches cover portions of the area of interest. The potential to map an area and to identify snow patches and their thickness profile is a complementary capability of the GPR that should be explored. Early work with GPR showed its ability to map stratigraphy on soil ${ }^{8}$ and ice.

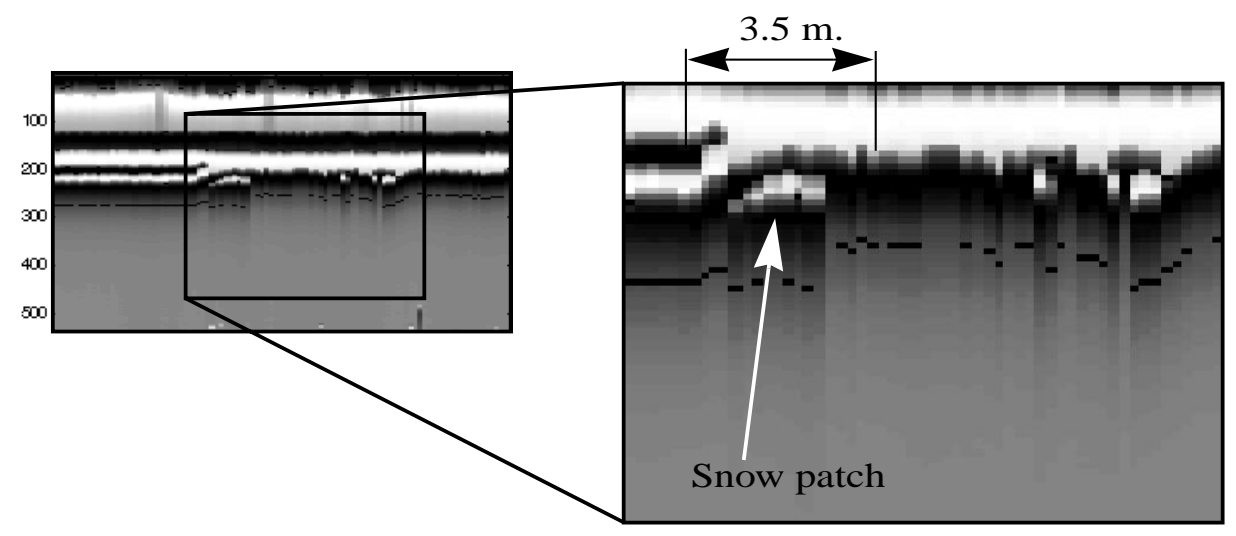

FIGURE 5. Ice/snow boundary as detected by the GPR sensor

A blue ice area with snow patches was selected for this experiment. The snow would reach $30 \mathrm{~cm}$ height in the thickest part of the patch. The antenna was dragged slowly over the ice, then over the snow patch and finally over the ice again. The results are shown in Figure 5. Additional testing is necessary to understand how the detection of buried objects is effected by their existence in the gap between the ice and the snow.

\section{DATA ENHANCEMENT}

The data analysis involves data filtering and profile clarification through vertical and horizontal filtering and energy migration $^{2}$. These techniques aim for better visualization of the relevant features. The vertical filtering uses a discrete low pass filter applied to each scan to attenuate noise at frequencies above the pulse frequency content. The horizontal filtering uses a high pass filter applied over the elements at the same depth of all scans to remove the constant horizontal bands. These constant bands correspond to external factors as the coupling between the antenna and the surface. There are horizontal features that are inherent to the sensor as internal noise or "ringing" that also appear as bands. They are removed by the use of this high pass horizontal filters.

Figure 6 shows an example of data enhancement by horizontal filtering. As a result, the target (A) is more visible and another target (B) is visible under simple inspection of the image. Additionally, the surface coupling band disappears. Note that the two strong signals at both sides of the images correspond to the end marking metal bars. 

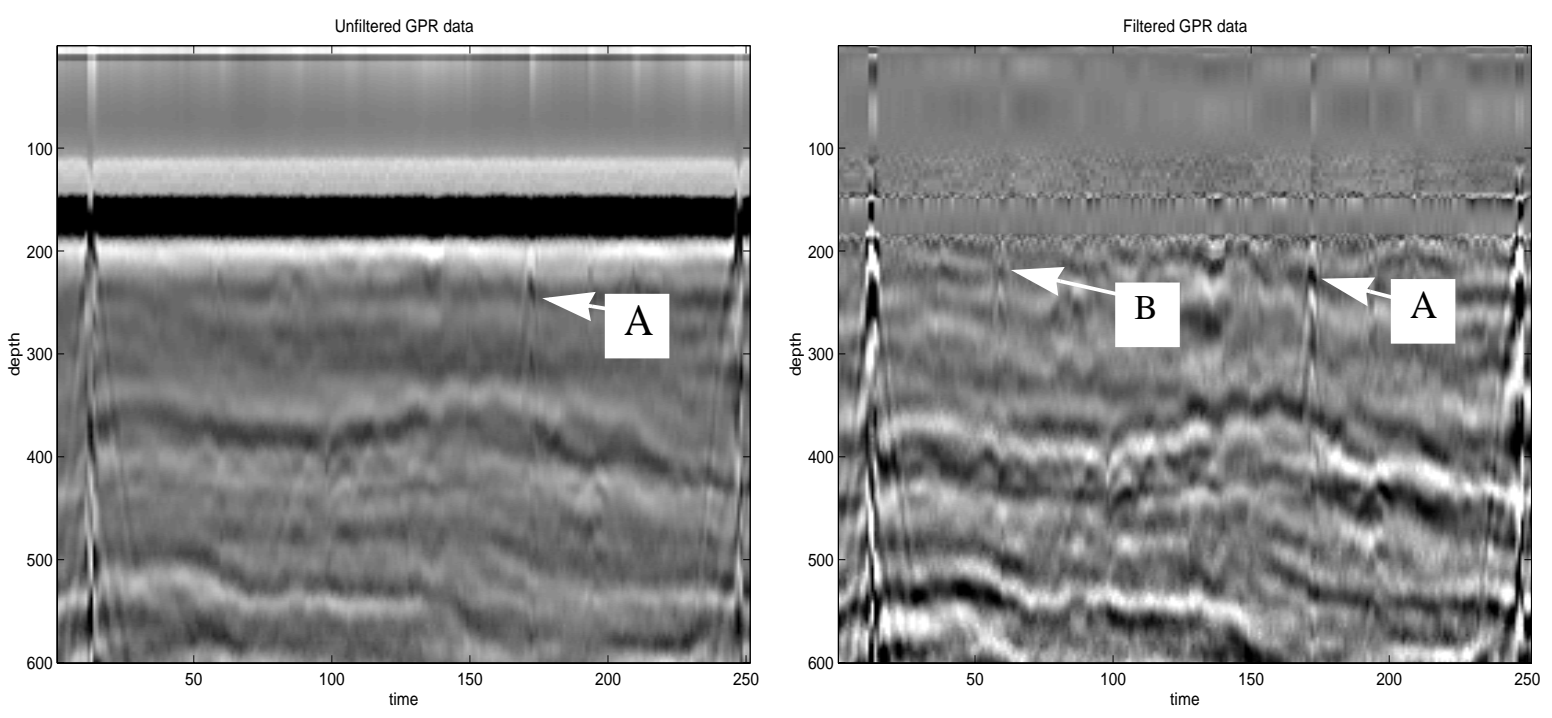

FIGURE 6. Enhancement by horizontal filtering.

The next step in development is a migration process that sharpens the radar profiles ${ }^{2}$. The signature of rocks appears as a peak in the data. Adaptive threshold applied to the profiles is a simple and effective technique to isolate those strong signals. Averaging the values gives a better position of the object with respect to the horizontal position. We are exploring correlation techniques for the automatic detection of crevasses and cracks. To improve the early detection, we intend to model the interaction of the wide beam with the vertical features to develop a technique that warns about a subsurface void that could endanger a robot.

\section{CONCLUSION}

A successful deployment of a GPR produced field data in Antarctica. The sensor demonstrated detection of medium size rocks $(>5 \mathrm{~cm}$.) in shallow depths $(<50 \mathrm{~cm}$.). However, the frequency $(500 \mathrm{MHz})$ proved to be too low to allow for detection of small objects. A radar sensor with higher frequencies (1 to $10 \mathrm{GHz}$ ) would be more appropriate for this task. Detection of objects between the ice and snow layers is still unclear. Further testing is required to solve this question.

Early void detection is a powerful feature when used to safeguard the robot from hidden obstacles and dangers. Better understanding of the particulars of the response of GPR to voids is necessary to avoid false positives caused by buried objects. The use of the GPR for safeguarding purpose requires design for deployment of the antenna ahead of the robot. Look-ahead ability might be compromised by change in GPR frequency.

It is likely that meteorites are located under seasonal snow drifts. Detection of seasonal snow drift patches is important to assist human search in areas of meteorite concentration. Snow/ice GPR stratigraphy proved detection and profiling of snow patches. 
We are in the process of automating this detection process. The prototypes and the sensor will be integrated on a robot for field validation and tested on the icefield in the next Antarctic season, as shown in Figure 7.

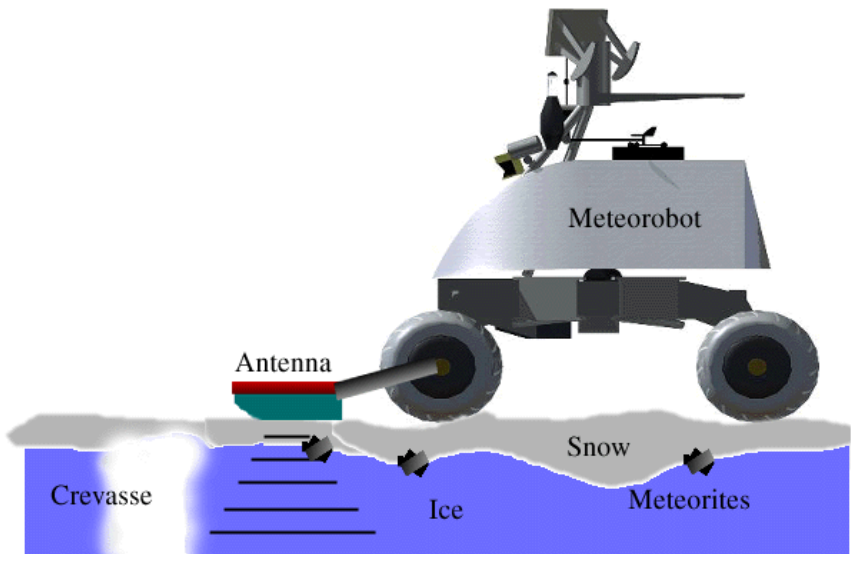

FIGURE 7. Planned use of the sensor for safeguarding and meteorite detection on a meteorobot.

\section{ACKNOWLEDGEMENTS}

This work was supported by the Robotic Antarctic Meteorite Search program. The program is funded by the Telerobotics Program UPN 632-40-42 of NASA's Office of Space Science Advanced Technology \& Mission Studies Division.

\section{REFERENCES}

1. P. Lee et al., "Search for meteorites in the Patriot Hills Area, Ellsworth Mountains, West Antarctica", Proceedings Meteoritical Society Meeting, Dublin, Ireland 27-31 July, 1998.

2. H. Herman, "Robotics subsurface mapping using ground penetrating radar", Carnegie Mellon University, School of Computer Science Technical Report CMU-RI-TR-97-19, 1997.

3. S. Arcone, "High Resolution of glacial ice stratigraphy: A ground penetrating radar study of Pegasus Runway, McMurdo Station, Antarctica". Geophysics, Vol. 61, No. 6 (Dec-Nov 1996), p 1653-1663.

4. A. Dellaney, S. Arcone, "Detection of crevasses near McMurdo Station, Antarctica with airborne short pulse radar", Cold Regions Research \& Engineering Laboratory Special Report 95-7.

5. S. Arcone, A Delaney, G. Blaisdell, "Airborne radar crevasse detection along the proposed south pole inland traverse", Proceedings 7th Annual SCALOP Symposium, Cambridge, England, Aug 5-9,1997.

6. A. Casas et al. "Detecting karstic cavities with ground penetrating radar at different geological environments in spain", Proceedings of the 6th International Conference of Ground Penetrating Radar, Sandai, Japan, Sept. 30-Oct 3, 1996.

7. V. Zolotarev, "Detection of cavities in dolomite and profiling of alluvial deposits using GPR in Lithuania", Proceedings of the 6th International Conference of Ground Penetrating Radar, Sandai, Japan, Sept. 30-Oct 3, 1996

8. J. Davis and A. Annan, "Ground penetrating radar for high resolution mapping of soil and rock stratigraphy”. Geophysical Prospecting 37, 521-551, 1989 